\title{
A Fractographic Analysis of Additively Manufactured Ti6Al4V by Electron Beam Melting: Effects of Powder Reuse
}

\author{
R. Schur $\cdot$ S. Ghods $\cdot$ E. Schultz $\cdot$ C. Wisdom $\cdot$ R. Pahuja $\cdot$ \\ A. Montelione $\cdot$ D. Arola $\cdot$ M. Ramulu
}

Submitted: 25 November 2019/in revised form: 28 March 2020/Published online: 20 May 2020

(C) ASM International 2020

\begin{abstract}
Metal additive manufacturing (AM) is being rapidly adopted in the aerospace and biomedical industries. Powder bed fusion AM processes are leading this trend. To maximize process economy, excess "unmelted" powder retrieved from the build chamber is used in subsequent build cycles. The metal properties and component reliability could undergo degradation with powder reuse. This study investigates the effects of powder reuse on fracture surface characteristics of Ti6Al4V specimens fabricated by electron beam melting AM over 30 sequential build cycles. Optical microscopy and scanning electron microscopy were used to evaluate the changes in fracture surface features of tensile failures with powder reuse. Macroscopically, slant fractures were most common in early builds, which transitioned to orthogonal fracture surfaces with poorly defined shear lips with increasing reuse. Regardless of the build number, the fracture origins were consistently from the as-built surfaces. Microscopically, ductile features such as micro-void coalescence were evident throughout the 30 build cycles. However, increasing flute content with reuse suggests that rising oxygen levels causes solution strengthening and limits the participation of active slip systems. These results highlight the
\end{abstract}

R. Schur $\cdot$ S. Ghods $\cdot$ E. Schultz $\cdot$ C. Wisdom $\cdot$ A. Montelione - D. Arola

Department of Materials Science and Engineering, University of Washington, Seattle, WA, USA

R. Pahuja · D. Arola · M. Ramulu ( $₫)$

Department of Mechanical Engineering, University of

Washington, Box 352600, Seattle, WA 98195-2120, USA

e-mail: ramulum@uw.edu importance of surface roughness and powder oxidation to metal performance in AM, and the evolution of fractographic features with powder reuse.

Keywords Additive manufacturing - Electron beam . Fractography $\cdot$ Powder bed $\cdot$ Reuse $\cdot$ Titanium

\section{Introduction}

In the last several decades, metal additive manufacturing (AM) has rapidly expanded from a concept process involved in developing prototypes to a viable method of product manufacturing [1]. Commercial systems are now available from various manufacturers and they accommodate a wide range of metals and alloys [2]. In response, metal AM has been rapidly adopted by a number of industries, and the most impressive advancements are taking place in the aerospace and the biomedical industry [3]. During 2010-2015, the value of AM rose by 30\%, with no sign of slowing [3].

AM provides several benefits over conventional methods of manufacturing, and in particular with respect to subtractive techniques. One of the most commonly cited advantages of AM overall is the geometric design freedom. Indeed, metal AM enables the creation of parts that are either impossible or too expensive to create through conventional means [4]. Topology optimization is also possible, which can significantly reduce the weight and material use of certain manufactured components [3]. This capability enables extremely low "buy-to-fly" ratios. In addition, whereas conventional manufacturing techniques such as casting typically exhibit a decreasing cost per unit part due to mold or tooling needs, the cost per part in metal 
AM remains approximately constant regardless of the number of parts built [4]. Thus, AM is suitable for a range of production scenarios, including small production runs and customized parts.

In industries such as aerospace that are producing components that are important to public safety, a certification is required before they can be placed on aircraft. Clearly, the need increases in stress-critical applications. But a significant amount of work is required before a clear path to component certification can be achieved. Most important in this pursuit is the development of processstructure-property relationships that help to establish confidence in the process and components $[5,6]$. Comprehensive models of the AM process are needed, which are supported by a wide selection of characterization techniques, to mitigate the need for individual qualification of every part produced. Requiring individual or $100 \%$ part certification could be the death of metal AM in aerospace.

In metal powder bed fusion (PBF) AM, only a portion of the metal powder within the build space undergoes fusion to form the printed parts. Owing to the high cost of AM feedstock in metal PBF processes, there is substantial interest in reusing the remaining powder that did not undergo melting. However, this powder has undergone thermal cycling within the build chamber and, in the case of electron beam melting (EBM) systems, has experienced mechanical damage as a consequence of the powder recovery process. As such, the issue of certification becomes increasingly complicated when considering powder reuse. A number of studies have attempted to address these concerns by examining the effects of powder damage on the printed parts [7-17]. Those focused on the use of Ti6Al4V powder have reported that there are significant changes in powder quality with reuse [8, 9, 11, 17-19]. Mostly commonly addressed are powder chemistry and its flowability, and mechanical properties of the printed parts. While valuable, an evaluation detailing the fractographic features of AM metals and the contributions of powder reuse to the tensile fracture characteristics has not been reported.

As metal AM becomes increasingly viable for stresscritical components and related applications, a deeper understanding of the predominant defects contributing to component failures and their fracture origins will be needed. Considering that powder reuse and the consequent changes in composition can cause a transition in failure modes, evaluations that address failure mechanisms become even more important. In the present experimental investigation, a fractographic analysis of tensile specimens developed by EBM of Ti6Al4V powder was conducted in a program that consisted of reusing powder in 30 sequential build cycles [18]. The objective of this investigation was to detail the fracture origins in tensile failures and the changes in fracture surface characteristics as a function of powder reuse.

\section{Materials and Methods}

The feedstock materials used in the present study consisted of $50 \mathrm{~kg}$ of Grade $5 \mathrm{Ti} 6 \mathrm{Al} 4 \mathrm{~V}$ gas atomized powder, which was acquired and certified by Arcam (Arcam: Batch P1303, Part \#430944) to be compliant with ASTM F2924-14 [20]. The powder was handled according to the manufacturer's recommendation in all aspects of the investigation.

The investigation consisted of 30 total build cycles, which were conducted sequentially on approximately a weekly routine. Printing was performed on an Arcam A2X EBM system using the default processing parameters for Grade 5 Ti6Al4V. For convenience, the builds were designated in terms of the build number (b\#) ranging from b1 to b30. The first build (b1) was performed with virgin powder. After each build, the sintered powder was recovered using the Arcam Powder Recovery System (PRS), sieved, and mixed with the powder that remained in the hoppers, and then placed in the hoppers for the subsequent build. By this approach, each build after b1 contained an increasing volume fraction ratio of used powder. No additional virgin powder was added to the powder volume during the study.

Each build contained a set of standard tensile specimens, including six vertical (ZY by ISO/ASTM 52921:2013(E) [21]) and six horizontal (YZX) subsize rectangular specimens as per ASTM E8M [22]. Three each of the horizontal and vertical specimens were subjected to tensile testing from each build in the as-built condition (i.e., without postprocessing). Additional specimens from selected builds were subjected to surface milling and then tested to determine the contribution of the surface texture to the failure. The surface roughness of the samples was measured using a Keyence optical profilometer (Keyence VR3100). The milling process reduced the average surface roughness $\left(R_{\mathrm{a}}\right)$ of the as-built specimens from approximately $30-1.7 \mu \mathrm{m}$ post-machining. The location of specimens within the build space was not changed between builds to avoid variability. Further details of the experimental design and methods of evaluating the properties can be found in our previously reported work [18].

After completion of the tensile testing, the fracture surfaces were initially evaluated using a stereomicroscope (Olympus SZX16) over a range of magnification $(10 \times-$ $100 \times)$. Thereafter, the gauge section of the specimens from the selected builds was separated from the grip sections using diamond abrasive wheels on a water-cooled slowspeed saw. The specimens were then sonicated in isopropyl alcohol (IPA) and water for approximately $5 \mathrm{~min}$ each. 
Thereafter, the fracture surfaces were analyzed using scanning electron microscopy (SEM, Philips XL-30). Detailed in these analyses were the fracture modes, apparent defects, and the \% shear lip area. To quantify the $\%$ shear lip area of the fracture surfaces, a series of SEM micrographs were acquired with point of view parallel to the loading axis. They were then stitched together to detail the entire fracture surface. Commercial software (ImageJ 1.52a) was used to quantify the shear lip area of the fracture surface, as well as total fracture surface area. The ratio of these measurements was used to quantify the $\%$ shear lip area. Admittedly, this approach provides an estimate according to the use of $2 \mathrm{D}$ projections of the fracture surface, rather than 3D data. Nevertheless, this approach provides an effective manner for achieving relative measures and is useful for tracking the change in apparent ductility of the material with powder reuse.

Several of the fractured specimens were also metallographically prepared to evaluate the fracture surface plane relative to the grain boundaries. For this analysis, the selected specimens were mounted flat, polished, and etched to reveal the underlying microstructure and the path of fracture with respect to the grain boundaries. The samples were mounted in potting component using standard procedures on a Buehler Pneumet I hot press. They were then ground and polished with progressively finer $\mathrm{SiC}$ paper and diamond suspensions. Finally, an attack polish was used to achieve a mirror finish followed by etching with Kroll's Reagent for approximately $15 \mathrm{~s}$.

\section{Results}

The fracture surfaces of selected representative tensile specimens are shown in Fig. 1. Specifically, tensile specimens obtained with vertical build direction from b5 and b30 are shown in Fig. 1a and b, respectively. Similarly, representative specimens obtained with the horizontal build direction from prints b1 and b30 are shown in Fig. 1c and $\mathrm{d}$, respectively. The fracture origin for each specimen is indicated with a pointer. There are lack-of-fusion (LOF) voids evident on the fracture surfaces of the horizontal specimens that are large enough to be seen at this low magnification. The larger voids resulting from LOF are highlighted by arrows. In addition, the build direction is noted on the side of each specimen for clarity. As evident from these figures, the fracture origins of both the horizontal and vertical specimens were identified at the surface of the specimens, as indicated by the pointers in Fig. 1 . This was consistent among all of the as-built tensile specimens evaluated without post-processing. Fracture origins of approximately $90 \%$ of the horizontal specimens were located at the edge of the specimens that was the last printed layer (farthest from build plate) of material in the parts. There was no change apparent in this phenomenon with build number. In contrast, although fracture initiated at the surface of the vertical specimens, they did not exhibit a preferential location of failure along the outer perimeter.

Figure 2a presents results of the \% shear lip area measurements for the fracture surfaces from selected builds. With the exception of a few specimens that failed to print, three horizontal and three vertical specimens were analyzed for each selected build. According to the distribution of the data, there is a progressive decrease in \% shear lip area on the fracture surfaces. In the early builds (b1-b5), the fracture surfaces are dominated by shear regardless of the build direction. However, in the builds involving heavily reused powder (b25-b30) the \% shear lip area decreased to below $50 \%$ for both build directions. Examples of horizontal fracture profiles from builds 1,15 , and 30 are shown in Fig. 2b. The profile views clearly illustrate the change in the fracture surfaces with increasing powder reuse as denoted quantitatively by the reduction in $\%$ shear lip area.
Fig. 1 Tensile specimen fracture surfaces of representative as-built specimens. (a) Vertical specimens of b5; (b) vertical specimens of heavily reused powder from b30; (c) horizontal specimens from b1; and (d) horizontal specimens from b30. The fracture origin for each is indicated with a pointer, and lack-of-fusion voids are highlighted by arrows. Build direction is noted on the side of each specimen
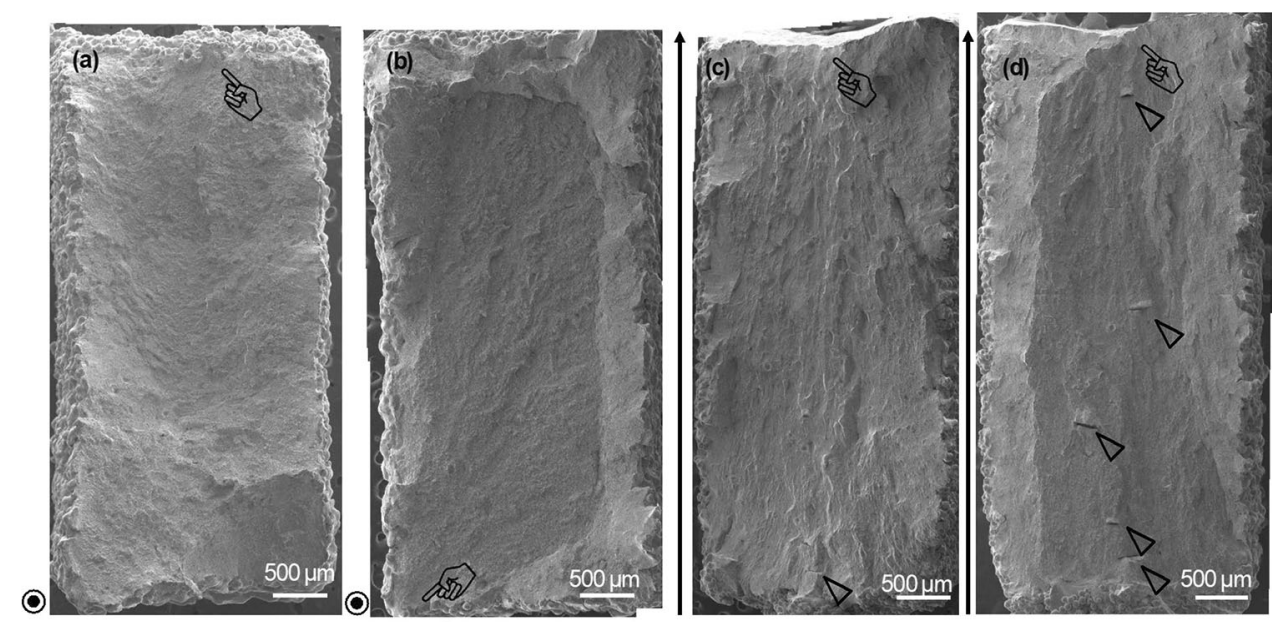
Fig. 2 Percentage of shear lip area occupying the total fracture surface as a function of the build cycle and powder reuse. Data points with a star only represent two samples. Representative fracture profiles of horizontal specimen from $b 1, b 15$, and $b 30$ are also shown
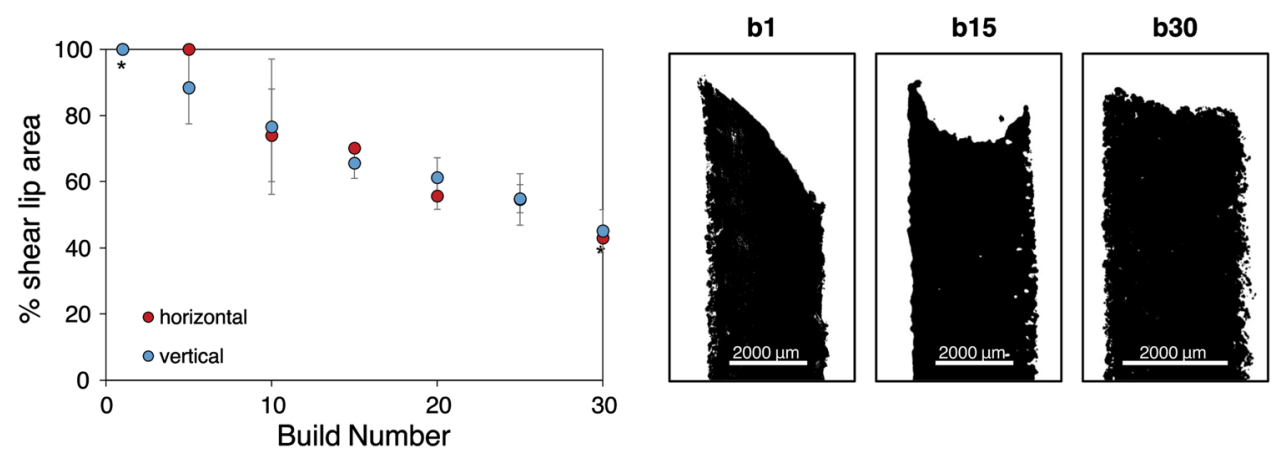

Fig. 3 Fracture surfaces of representative specimens in the post-machined condition.

Shown are vertical specimens of (a) lightly reused from b6 and (b) heavily reused powder of b26, as well as horizontal specimens from (c) lightly reused of b6 and (d) heavily reused powder of $b 26$. The fracture origins are denoted with a pointer, and the build direction is noted on the side of each specimen with an arrow

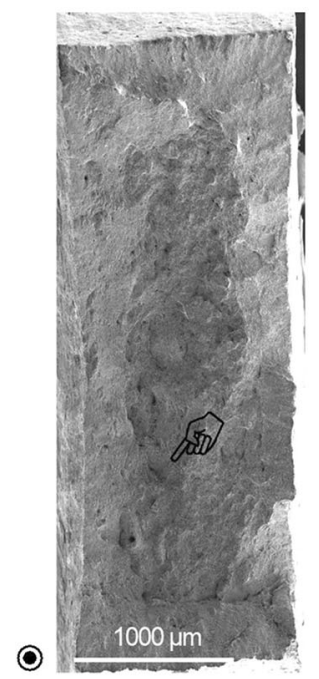

(a)

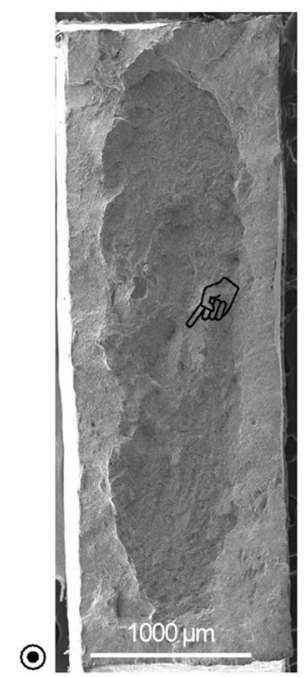

(b)

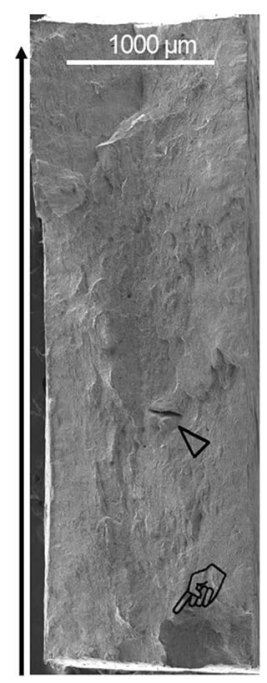

(c)

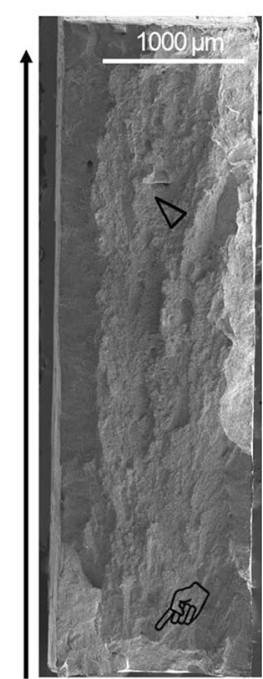

(d)
Due to the influence of surface roughness on the initiation of fracture (e.g., Fig. 1), a group of vertical and horizontal build specimens were subjected to surface milling to improve the surface texture. The fracture surfaces of selected tensile specimens from this group are shown in Fig. 3. Specifically, vertical specimens from b6 and b26 are shown in Fig. 3a and b, respectively. They represent builds conducted with lightly and heavily reused powder, respectively. Similarly, horizontal specimens from b6 and b26 are shown in Fig. 3c and d, respectively. The fracture origins are denoted with a pointer where discernible and the build direction is noted on the side of each specimen. Contrary to the specimens evaluated in the as-built condition, the failure origins of the machined specimens were identified on the interior of all these specimens. In general, they exhibited a more characteristic cup-cone fracture surface in comparison with the fracture surface morphology of those that were not machined.

Details of the fracture surfaces are shown under a higher degree of magnification in Fig. 4. As-built horizontally and vertically oriented specimens are shown from b5 in Fig. 4a and $b$, respectively, and as-built horizontally and vertically oriented specimens from b26 are shown in Fig. 4c and d, respectively. Micro-void coalescence is clearly evident in the fracture surfaces of all specimens, regardless of build direction, surface preparation, or degree of powder reuse. The size of dimpling varied considerably across the surface of each specimen.

In addition to the appearance of micro-void coalescence, many of the fracture surfaces also exhibited small colonies of fracture flutes, particularly after build 12. Micrographs obtained from SEM analysis that detail flutes identified on the fracture surface of representative specimens are shown in Fig. 5. Specifically, examples from horizontal specimens from b12, b26, and b30 are shown in Fig. 5a through c, respectively. The dimension of the flutes varied in size, but appeared to be less than $100 \mu \mathrm{m}$ in length and significantly less than that in width. In addition, although no quantitative measures of flute area were obtained, they appeared more frequently and in greater colony size in horizontal specimens that were built with more heavily reused powder (b25-b30). Furthermore, the orientation of the flutes varied 
Fig. 4 Examples of micro-void coalescence in the fracture surfaces of specimens for specific build number and orientation. (a) b5-horizontal, (b) b5-vertical, (c) b26horizontal, and (d) b26vertical specimens
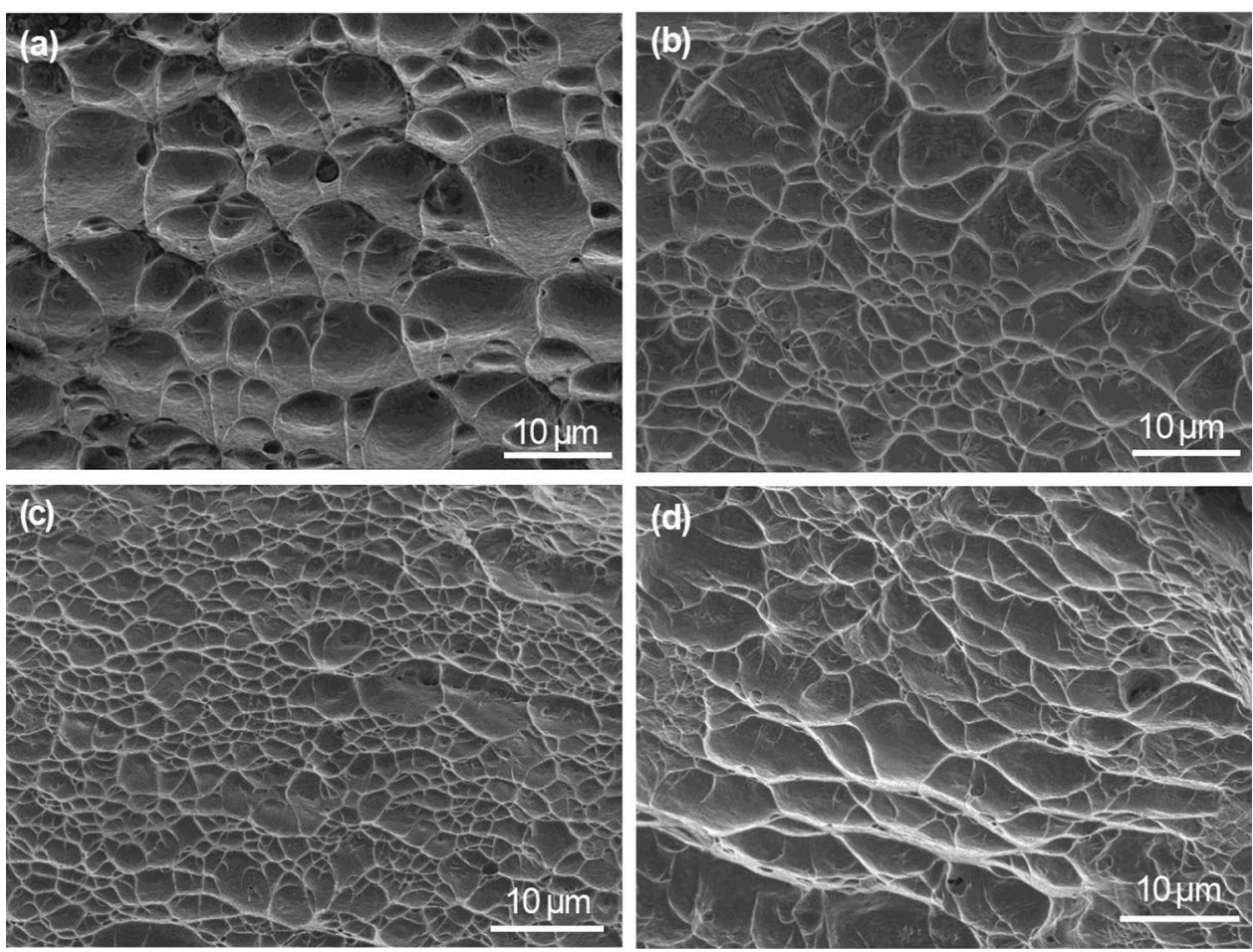
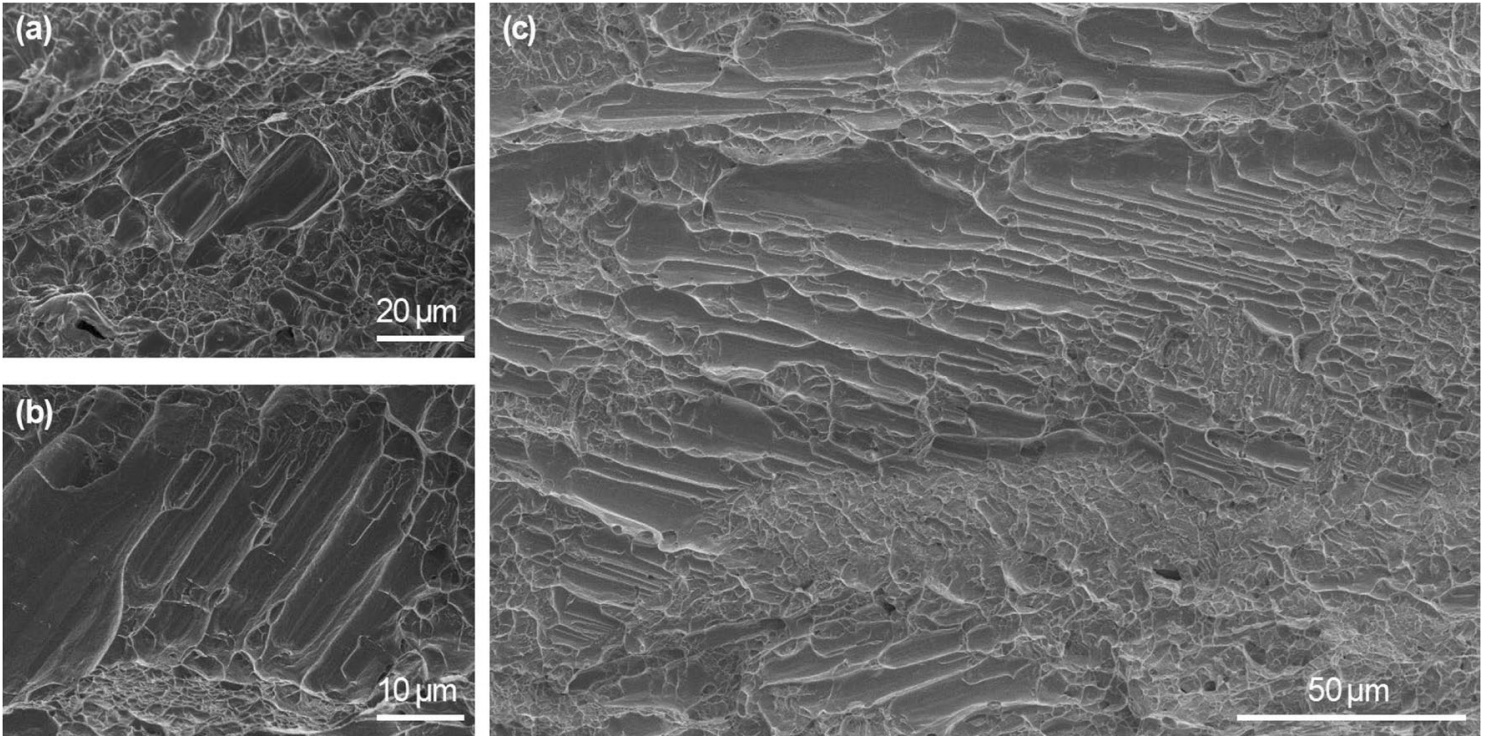

Fig. 5 Flutes identified in the fracture surface of representative specimens with the largest flutes indicated by arrows. (a) b12, (b) b26, and (c) b30

across the fracture surfaces and did not appear to follow any pattern.

The microstructure of many of the tensile specimens was revealed through mounting, polishing, and etching in Kroll's reagent. A typical micrograph is presented in Fig. 6 of a vertical specimen as a reference for discussion of the contribution of the microstructure to fracture. The microstructure consists of columnar prior $\beta$-grains oriented parallel to the build direction, as noted by the arrows in the figure. The interior of these prior $\beta$-grains contains a typical basket-weave structure comprised of $\beta$-ribs within an $\alpha$-matrix. This microstructure is representative of both the vertical and horizontal build directions; for both specimen orientations, the prior $\beta$-grains follow the build direction. Hence, the only difference between the vertical and horizontal tensile specimens is the orientation of these features 
Vertical Tensile

Specimen Loading

Direction

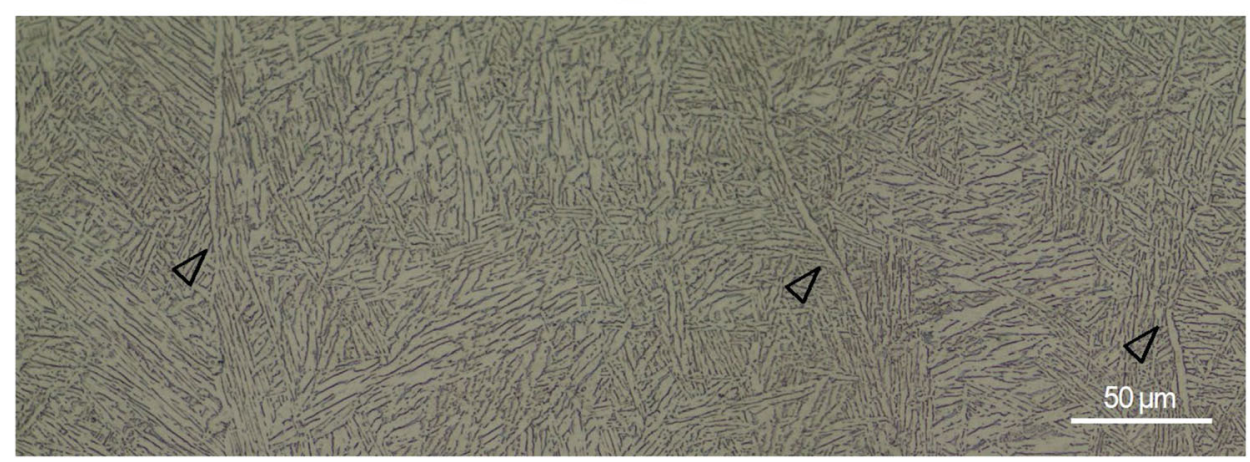

Horizontal Tensile Specimen Loading Direction

Fig. 6 Typical microstructure of a vertical oriented tensile specimen, consisting of elongated prior $\beta$-grains parallel to the build direction (denoted by arrows) and a basket-weave structure within. The direction of loading with respect to the microstructure is shown for the horizontal and vertical build directions
Fig. 7 Etched horizontal specimens from $b 1$ and $b 30$ adjacent to the fracture surface. (a) and (b) Show that the cracks propagated along prior $\beta$-grain boundaries in $\mathrm{b} 1$ and $\mathrm{b} 30$, respectively, and (c) through basket-weave colonies. Prior $\beta$ grains are highlighted by arrows
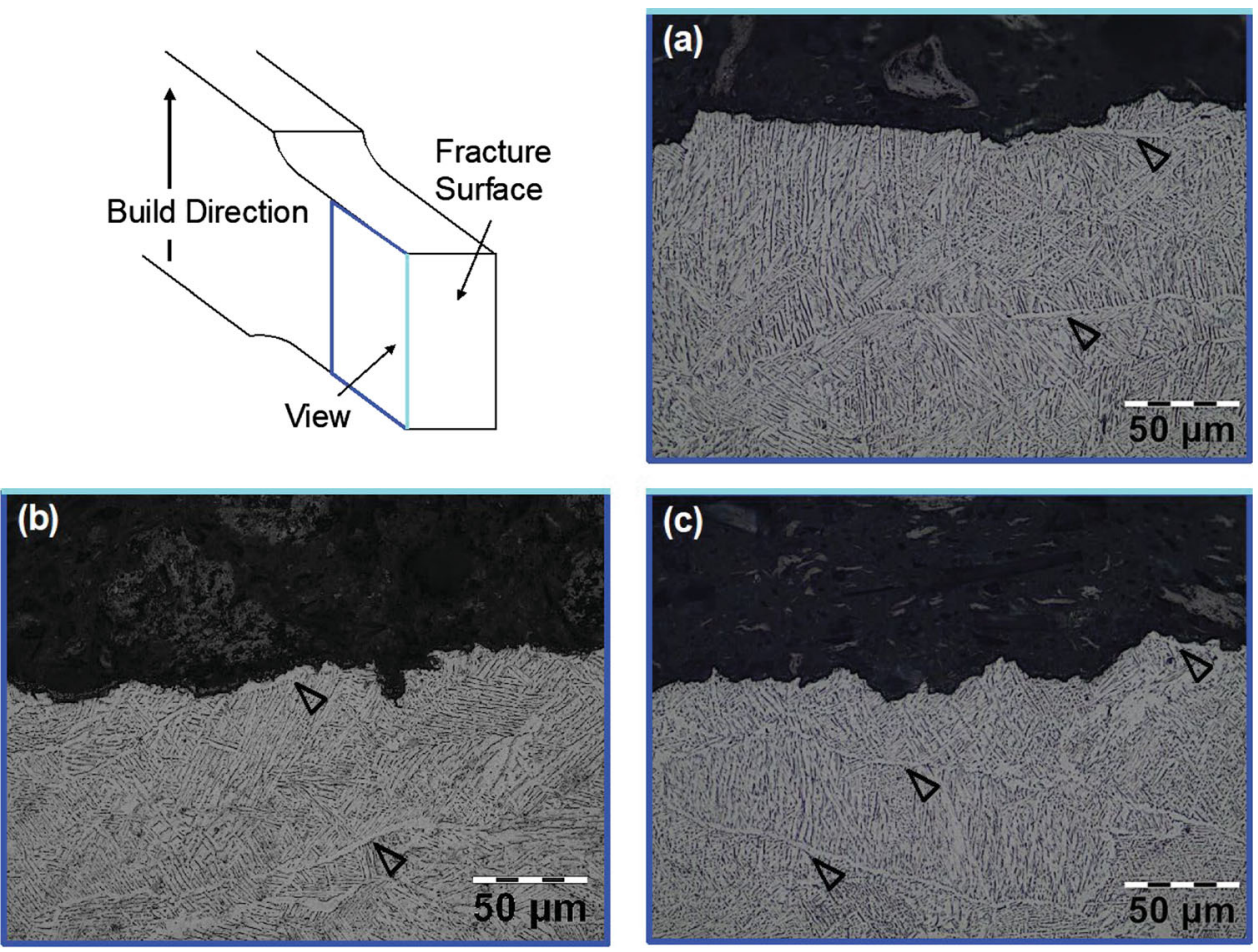

with respect to the principle loading direction. In the vertically oriented samples, the prior $\beta$-grains are parallel to the loading direction, whereas in the horizontal samples they are perpendicular to it. These loading directions are annotated in the figure for clarity.

Additionally, several of the fracture specimens were mounted, polished, and etched in Kroll's to reveal the location of the fracture plane relative to the microstructure. Two regions of a mounted specimen with horizontal build direction from b1 are shown in Fig. 7, as well as a region from a horizontal sample from b30. Figure $7 \mathrm{a}$ and $\mathrm{b}$ details portions of the fracture surface of $b 1$ and b30, respectively, in which the path of fracture coincided with a prior $\beta$-grain boundary as noted by the arrow, indicating intergranular fracture. However, the plane of fracture in Fig. 7c corresponding to another region of the fracture surface from the b1 horizontal specimen does not coincide with a prior $\beta$ - 
Table 1 The average mechanical properties for as-built horizontal and vertical specimens for selected builds

\begin{tabular}{|c|c|c|c|c|c|c|c|c|}
\hline & \multicolumn{2}{|c|}{ Elastic modulus (GPa) } & \multicolumn{2}{|c|}{ Yield strength (MPa) } & \multicolumn{2}{|c|}{ UTS (MPa) } & \multicolumn{2}{|c|}{$\%$ Elongation } \\
\hline & Horizontal & Vertical & Horizontal & Vertical & Horizontal & Vertical & Horizontal & Vertical \\
\hline b1 & $92.7 \pm 2.5$ & $98.9 \pm 3.2$ & $739 \pm 2.7$ & $733 \pm 20.7$ & $857 \pm 10.4$ & $859 \pm 20.5$ & $9.28 \pm 1.06$ & $12.2 \pm 0.42$ \\
\hline b5 & $108 \pm 0.3$ & $109 \pm 2.5$ & $853 \pm 8.3$ & $814 \pm 6.1$ & $982 \pm 7.8$ & $942 \pm 7.8$ & $8.37 \pm 0.29$ & $10.4 \pm 0.48$ \\
\hline b10 & $108 \pm 7$ & $116 \pm 3.4$ & $837 \pm 2.6$ & $838 \pm 9.5$ & $940 \pm 7.6$ & $924 \pm 16.6$ & $6.38 \pm 0.88$ & $6.91 \pm 0.27$ \\
\hline b15 & $107 \pm 5.9$ & $114 \pm 7.8$ & $860 \pm 1.7$ & $866 \pm 3.6$ & $970 \pm 11.2$ & $999 \pm 14.7$ & $6.28 \pm 0.37$ & $9.71 \pm 0.46$ \\
\hline $\mathrm{b} 20$ & $102 \pm 0.8$ & $113 \pm 5.9$ & $927 \pm 18.9$ & $939 \pm 7.5$ & $1010 \pm 21.8$ & $1070 \pm 11.3$ & $4.30 \pm 0.25$ & $9.18 \pm 0.99$ \\
\hline $\mathrm{b} 25$ & $97.4 \pm 0$ & $109 \pm 2.3$ & $1020 \pm 0$ & $973 \pm 16$ & $1100 \pm 0$ & $1100 \pm 11.5$ & $3.61 \pm 0$ & $7.77 \pm 0.41$ \\
\hline b30 & $109 \pm 1.6$ & $109 \pm 6.0$ & $1050 \pm 5.3$ & $1000 \pm 18.5$ & $1120 \pm 8.7$ & $1130 \pm 188.6$ & $3.53 \pm 0.26$ & $7.34 \pm 0.33$ \\
\hline
\end{tabular}

Properties include the elastic modulus, yield strength, ultimate tensile strength (UTS), and \% elongation at failure

grain boundary. Instead, it follows an intragranular path along the basket-weave structure of the $\alpha$-lath and $\beta$-ribs.

A summary of the results from tensile testing of as-built specimen is presented in Table 1 . This table contains the averages of the elastic modulus, yield strength, ultimate tensile strength (UTS), and \% elongation at failure for specific builds and for both build directions. The builds represented in this table correspond to those with corresponding \% shear lip area measurements (Fig. 2). The trends indicate rising yield strength and UTS with powder reuse, whereas the \% elongation decreases. The elastic modulus does not undergo substantial changes with reuse. Additionally, there is a significant degree of anisotropy in the $\%$ elongation results. The vertical specimens exhibit a greater degree of ductility than those with horizontal orientation.

\section{Discussion}

Powder reuse in metal AM by $\mathrm{PBF}$ is of substantial importance, and a number of earlier studies have been reported on this topic for the EBM process [8-11, 19]. In general, prior efforts concerning AM of Ti6Al4V have focused primarily on the microstructure and mechanical behavior of the metal as a function of reuse [8-10]. In comparison, changes in the features of the fracture surfaces with powder reuse and the origins of failure, which are critical to understanding metal quality, have largely been neglected in the literature. Only a few prior studies have addressed this topic in some detail, but were primarily focused on fatigue [7, 10]. The study by Popov et al. [10] does present some useful details of a fractographic analysis of tensile specimens that were built with virgin and reused powder. Nevertheless, a greater degree of depth on this subject is required to provide information that can be used to guide future forensic studies involving metal AM components.
According to results of the evaluation targeting the fracture origins, fracture of all the tensile specimens evaluated in the as-built condition initiated from the surface, as shown in Fig. 1a-d. There was no apparent influence of the extent of powder reuse on the origin of failure. The failures initiated at the surface due to the large surface stress concentrations posed by the surface roughness. For EBM AM of Ti6Al4V, Wang et al. [23] found that the average surface roughness $\left(R_{\mathrm{a}}\right)$ can reach as high as $34 \mu \mathrm{m}$ for the horizontal build direction and up to $40 \mu \mathrm{m}$ for the vertical direction. Clearly, the surface stress concentrations caused by the surface roughness were more detrimental to the initiation of failure than the LOF voids that were identified within the interior. Hence, the surface quality is a plausible contributor to failures of metal AM components used in the "as-built" condition and should be considered in forensic investigations.

To assess the changes in fracture origin if post-processing is applied to remove the "as-printed" rough surfaces, selected tensile specimens were obtained over the powder reuse study and machined to remove the surface texture resulting from AM. Interestingly, the fracture origins of all the machined samples were located within the interior (Fig. 2) and not at the build surface, regardless of the build direction or the extent of powder reuse. Therefore, the surface roughness of specimens in the as-built condition resulted in the initiation of cracks, which appeared to have caused the material to fail in a more brittle manner macroscopically. These results are in agreement with the findings of others as well [24]. Hence, the apparent ductility of Ti6Al4V components produced by EBM metal AM and applied in the as-built condition can be limited by the large surface roughness.

One unique and unexpected finding involved the fracture origins of the horizontal specimens. Contrary to the random location of failure along the perimeter for those with vertical build orientation, the origin of failure for the specimens with horizontal build orientation was usually at the top surface, corresponding to the final layers of printed 
material. This surface of the horizontal specimens has a lower surface roughness than the two vertical surfaces, and especially with respect to that of the bottom surface where support structures were removed [23]. A plausible explanation of the horizontal specimen failure origins is the unique microstructure at this top surface. Decomposition of $\alpha^{\prime}$-martensite to the $\alpha-\beta$ structure of Ti6Al4V, which occupies the majority of the specimen volume, can become restricted within the final top layers of a part due to the reduced thermal energy to drive this process. Freezing of the top layers is not followed by the deposition of additional thermal energy in subsequent build layers, thereby resulting in retained $\alpha^{\prime}$ at the surface [25]. Since $\alpha^{\prime}$ martensite is brittle with regard to the $\alpha-\beta$ structure of Ti6Al4V, the surfaces could become sensitized to crack initiation. A cursory microstructural analysis was performed on the specimens, and it was found that there was a reduction in $\alpha$-lath thickness in the final printed layers of these horizontal specimens. Some of these laths exhibited aspect ratios high enough to be defined as martensite [26].

To complement the assessment of fracture origins performed in this investigation, the fracture surfaces from selected b1 and b30 horizontal and vertical tensile specimens were mounted, polished, and etched to identify the path of fracture relative to the microstructure. In the horizontal specimens, the path of fracture either followed the prior $\beta$-grain boundaries (Fig. 7a and b) when they were present, or the grain boundaries in the Widmanstätten structure (Fig. 7c). Note that the exact fracture path was dependent on the fracture origin from the surface and its coincidence with prior $\beta$-grain boundaries. Previous studies have shown that there is a fine $\alpha$-layer surrounding the prior $\beta$-grains [27] and that this $\alpha$-rich phase provides a path of least resistance for crack propagation when loading is applied perpendicular to the prior $\beta$-grain interfaces. Indeed, due to the columnar grain structure oriented perpendicular to the loading direction of the horizontal tensile specimens shown in Fig. 6, these boundaries were appropriately oriented for intergranular fracture. Conversely, the prior $\beta$-grains in the vertical specimens are oriented parallel to the loading direction, which precluded the path of fracture from continuing in an intergranular manner along the prior $\beta$-grain boundaries. Indeed, the vertically oriented specimens exhibited higher \% elongation than those with horizontal orientation as given in Table 1. Therefore, the grain structure and orientation of columnar grains relative to the fracture plane should be examined in an evaluation of fractures in Ti6Al4 $\mathrm{V}$ components produced by EBM.

Although the fracture origins did not change as a function of powder reuse, other aspects of the fracture surface did. One such feature was the percentage of shear lip area of the fracture surface. Shear lips are indicative of the mechanisms of failure and are nearly universally recognized as a sign of ductility. In general, failures with a higher \% shear lip area should be more ductile and vice versa. A quantification of the $\%$ shear lip area with powder reuse showed that the $\%$ shear lip area was consistent for both build directions. Furthermore, the shear lip area decreased significantly with powder reuse from approximately $100 \%$ with virgin powder (associated with single and double slant shear fractures) to less than $50 \%$ by b30 involving the heaviest degree of powder reuse. The reduction in shear lip area with powder reuse is consistent with the reduction in \% elongation of the Ti6Al4 V obtained from results of mechanical testing, as given in Table 1. The oxygen content of the powder from this study was previously found to increase linearly with powder reuse [18], an effect that can cause severe embrittlement of the material [28]. This transition in material behavior was clearly evident from the fracture surface as well as through the decrease in shear lip area. The characteristic fracture profiles from specimens b1, b15, and b30 in Fig. 2 illustrate the change from pure slant fracture in b1 to nearly flat fracture surface orientations by b30.

When the fracture surfaces are viewed at higher magnification, the dominant feature is micro-void coalescence, as shown in Fig. 4. This is true regardless of build direction or powder age, indicating some degree of retained material ductility despite the macroscopic changes. The occurrence of brittle macroscopic features paired with ductile microvoid coalescence is well established for hexagonal closepacked (HCP) metals such as $\alpha$-titanium due to the limited slip systems present [29]. In addition, the size of the dimples was observed to vary within individual fracture surfaces. One possible explanation for this is an observed range of grain sizes. As previously reported, the size of microstructural features varied significantly within individual samples [18], both within layers and with build height. Given that dimples can form at grain boundaries [30], this could result in a wide distribution of dimple sizes within a single specimen.

At higher levels of magnification, fracture flutes became evident on the fracture surfaces of specimens from builds 12 and higher, as shown in Fig. 5. Flutes are elongated dimples that are common to HCP structures [31]; they are considered a ductile fracture surface feature [32]. In titanium alloys, they tend to form on $\{10 \overline{1} 0\}\langle 11 \overline{2} 0\rangle$ along $\alpha-\alpha$ or $\alpha-\beta$ boundaries and are also often formed in Widmanstätten microstructures due to the close packing of the $\alpha$ - and $\beta$-plates [33]. According to Nixon and Hawkins [34], the flutes can exhibit a variety of sizes, from single microns to approaching hundreds of microns. Furthermore, they can appear as an isolated flute or in colonies, both of which were observed in the fracture surfaces of the Ti6Al4 V specimens. They can also appear under a number 
of circumstances, including stress corrosion cracking, hydrogen and oxygen embrittlement, and fracture at low temperatures [33].

In a complementary assessment of the powder feedstock from this study and the changes with reuse, it was established that the oxygen content of the powder increased linearly with increasing build number [18]. By the first observation of flutes in specimens of b12, the oxygen content of the powder had surpassed $0.2 \mathrm{wt} . \%$ [18]. The progressive increase in oxygen content could limit the active slip systems in the HCP $\alpha$-phase of Ti6A4V, thereby encouraging the formation of flutes [33]. It appears from the fractographic evaluation that the oxygen content had manifested to a level by b12 that was sufficient to have impeded slip and cause a transition in the mechanisms of failure. Indeed, as the oxygen content continued to rise with further powder reuse [18], the flutes became more common and larger in size (Fig. 5a-c). Thus, oxygen content measurements should accompany fractographic analyses of Ti6Al4V component failures produced by PBF AM processes, especially if fracture flutes are identified.

Furthermore, while the flutes were parallel within colonies, the orientation of colonies did not appear to adhere to any pattern with respect to each other or the fracture surface. This is not unexpected, given that flutes have been shown to parallel the [0001] direction of the underlying $\alpha$-phase [32] and AM Ti6Al4V can form 12 distinct $\alpha$-variants during the $\beta$ - to $\alpha$-phase transformation [35]. This could give rise to a variety of flute orientations on a single fracture surface, which is consistent with our observations.

Results of this investigation have provided new understanding of the fracture surface characteristics of Ti6Al4V produced by EBM AM and the importance of powder reuse. The findings should serve as a reference in future studies reporting on the mechanisms of failure for metal AM components. Despite the merits of this work, there are some limitations that should be considered. Of course, the measurements of shear lip area were conducted based on a $2 \mathrm{D}$ evaluation of the fracture surfaces. That poses some limitations to accurately defining the exact shear lip area, but would not change the trends in behavior with powder reuse. In addition, oxygen content measurements were performed over the program from $\mathrm{b} 1$ to $\mathrm{b} 30$ for the powder, but not the built metal. Thus, although the cause for transition in failure mechanisms with an increase in powder reuse is assumed to be attributed to a rise in oxygen content, the actual changes in oxygen content in the printed metal are not known. Future work involving powder reuse in metal AM should evaluate the changes in composition of both powder and built metal. It should also be considered in a forensic evaluation of Ti6Al4V components produced by powder bed fusion AM.

\section{Conclusion}

A detailed experimental evaluation of the fracture surface characteristics of specimens obtained from metal additive manufacturing of Ti6Al4 V and subjected to uniaxial tension to failure was conducted. The specimens were built with horizontal and vertical directions and followed a printing routine that involved a total of 30 build cycles with powder reuse. It was found that the failure origins of specimens evaluated in the as-built condition were at the surface regardless of the build orientation. The surface roughness resulting from the electron beam melting process promoted large surface stress concentrations that served to facilitate the initiation of failure. There was no preferential location of failure in the specimens with vertical build direction, whereas failure of the horizontal specimens occurred at the last printed surface, likely due to the presence of finer and more brittle microstructure. The $\%$ shear lip area of the fracture surfaces decreased with increasing powder reuse, and there was no significant difference in the trends between the horizontal and vertical build directions. Microscopically, micro-void coalescence was evident on the fracture surfaces of all specimens and regardless of orientation or extent of powder reuse. Flutes were noted on the fracture surface of b12 and later with frequency increasing in later builds. The flutes appeared to develop from a reduction in slip in the HCP $\alpha$-phase due to increasing oxygen content of the material with powder reuse. The path of fracture involved both intergranular and intragranular planes. Due to the orientation of prior $\beta$ grains, fracture of the horizontal specimens preferentially followed these grain boundaries, whereas the part of fracture in the vertical specimens tended to follow grain boundaries in Widmanstätten colonies.

Acknowledgments This work was partially accomplished using facilities funded by the Joint Center for Deployment and Research in Earth Abundant Materials (JCDREAM) in Washington State. The authors also gratefully acknowledge support for this investigation from the Boeing Company through the Boeing Advanced Research Center.

\section{References}

1. T. DebRoy, H.L. Wei, J.S. Zuback, T. Mukherjee, J.W. Elmer, J.O. Milewski, A.M. Beese, A. Wilson-Heid, A. De, W. Zhang, Additive manufacturing of metallic components-process, structure and properties. Prog. Mater Sci. 92, 112-224 (2018). https://doi.org/10.1016/j.pmatsci.2017.10.001

2. N. Li, S. Huang, G. Zhang, R. Qin, W. Liu, H. Xiong, G. Shi, J. Blackburn, Progress in additive manufacturing on new materials: a review. J. Mater. Sci. Technol. 35, 242-269 (2019). https://doi. org/10.1016/j.jmst.2018.09.002

3. D.L. Bourell, Perspectives on additive manufacturing. Annu. Rev. Mater. Res. 46, 1-18 (2016). https://doi.org/10.1146/ annurev-matsci-070115-031606 
4. E. Atzeni, A. Salmi, Economics of additive manufacturing for end-usable metal parts. Int. J. Adv. Manuf. Technol. 62, 11471155 (2012). https://doi.org/10.1007/s00170-011-3878-1

5. M. Seifi, A. Salem, J. Beuth, O. Harrysson, J.J. Lewandowski, Overview of materials qualification needs for metal additive manufacturing. JOM 68, 747-764 (2016). https://doi.org/10. 1007/s11837-015-1810-0

6. M. Seifi, M. Gorelik, J. Waller, N. Hrabe, N. Shamsaei, S. Daniewicz, J.J. Lewandowski, Progress towards metal additive manufacturing standardization to support qualification and certification. JOM 69, 439-455 (2017). https://doi.org/10.1007/ s11837-017-2265-2

7. P.E. Carrion, A. Soltani-Tehrani, N. Phan, N. Shamsaei, Powder recycling effects on the tensile and fatigue behavior of additively manufactured Ti-6Al-4V parts. JOM 71, 963-973 (2019). https:// doi.org/10.1007/s11837-018-3248-7

8. H.P. Tang, M. Qian, N. Liu, X.Z. Zhang, G.Y. Yang, J. Wang, Effect of powder reuse times on additive manufacturing of Ti$6 \mathrm{Al}-4 \mathrm{~V}$ by selective electron beam melting. JOM 67, 555-563 (2015). https://doi.org/10.1007/s11837-015-1300-4

9. C. Wei, X. Ma, X. Yang, M. Zhou, C. Wang, Y. Zheng, W. Zhang, Z. Li, Microstructural and property evolution of Ti6Al4V powders with the number of usage in additive manufacturing by electron beam melting. Mater. Lett. 221, 111-114 (2018). https:// doi.org/10.1016/J.MATLET.2018.03.124

10. V.V. Popov, A. Katz-Demyanetz, A. Garkun, M. Bamberger, The effect of powder recycling on the mechanical properties and microstructure of electron beam melted Ti-6Al-4 V specimens. Addit. Manuf. 22, 834-843 (2018). https://doi.org/10.1016/J. ADDMA.2018.06.003

11. P. Nandwana, W.H. Peter, R.R. Dehoff, L.E. Lowe, M.M. Kirka, F. Medina, S.S. Babu, Recyclability study on Inconel 718 and Ti$6 \mathrm{Al}-4 \mathrm{~V}$ powders for use in electron beam melting. Metall. Mater. Trans. B 47B, 754-762 (2016). https://doi.org/10.1007/s11663015-0477-9

12. K.L. Terrassa, J.C. Haley, B.E. MacDonald, J.M. Schoenung, Reuse of powder feedstock for directed energy deposition. Powder Technol. 338, 819-829 (2018). https://doi.org/10.1016/J. POWTEC.2018.07.065

13. H. Asgari, C. Baxter, K. Hosseinkhani, M. Mohammadi, On microstructure and mechanical properties of additively manufactured AlSi10Mg_200C using recycled powder. Mater. Sci. Eng. A 707, 148-158 (2017). https://doi.org/10.1016/j.msea.2017.09.041

14. O.A. Quintana, J. Alvarez, R. Mcmillan, W. Tong, C. Tomonto, Effects of reusing Ti-6Al-4V powder in a selective laser melting additive system operated in an industrial setting. JOM 70, 18631869 (2018). https://doi.org/10.1007/s11837-018-3011-0

15. L.C. Ardila, F. Garciandia, J.B. González-Díaz, P. Álvarez, A. Echeverria, M.M. Petite, R. Deffley, J. Ochoa, Effect of IN718 recycled powder reuse on properties of parts manufactured by means of selective laser melting. Phys. Procedia 56, 99-107 (2014). https://doi.org/10.1016/j.phpro.2014.08.152

16. J. Nicolas, A. Bois-Brochu, C. Blais, Effect of oxygen content in new and reused powder on microstructural and mechanical properties of Ti6Al4V parts produced by directed energy deposition. Addit. Manuf. 23, 197-205 (2018). https://doi.org/10. 1016/j.addma.2018.08.011

17. Y. Sun, M. Aindow, R.J. Hebert, The effect of recycling on the oxygen distribution in Ti-6Al-4V powder for additive manufacturing. Mater. High Temp. 35, 217-224 (2018). https://doi.org/10. 1080/09603409.2017.1389133

18. S. Ghods, E. Schultz, C. Wisdom, R. Schur, R. Pahuja, A. Montelione, D. Arola, M. Ramulu, Electron beam additive manufacturing of Ti6Al4V: evolution of powder morphology and part microstructure with powder reuse. Materialia 9, 100631 (2020). https://doi.org/10.1016/j.mtla.2020.100631
19. V. Petrovic, R. Ninerola, Powder recyclability in electron beam melting for aeronautical use. Aircr Eng Aerosp Technol Int J 87, 147-155 (2015)

20. ASTM-International, ASTM F2924-14: Standard Specification for Additive Manufacturing Titanium-6 Aluminum-4 Vanadium with Powder Bed Fusion, pp. 1-9 (2014). https://doi.org/10.1520/ F2924-14.2

21. ISO/ASTM-International, ISO/ASTM 52921:2013(E) Standard Terminology for Additive Manufacturing-Coordinate Systems and Test Methodologies, pp. 1-13 (2019). www.astm.org. Accessed 21 Oct 2019

22. ASTM-International, ASTM Standard E8/E8M-16a: Standard Test Methods for Tension Testing of Metallic Materials, pp. 1-30 (2018)

23. P. Wang, W.J. Sin, M.L.S. Nai, J. Wei, P. Wang, W.J. Sin, M.L.S. Nai, J. Wei, Effects of processing parameters on surface roughness of additive manufactured Ti-6Al-4V via electron beam melting. Materials 10, 1-11 (2017). https://doi.org/10.3390/ ma10101121

24. W. Everhart, E. Sawyer, T. Neidt, J. Dinardo, B. Brown, The effect of surface finish on tensile behavior of additively manufactured tensile bars. J. Mater. Sci. 51, 3836-3845 (2016). https:// doi.org/10.1007/s10853-015-9702-9

25. H. Sharma, D. Parfitt, A.K. Syed, D. Wimpenny, E. Muzangaza, G. Baxter, B. Chen, A critical evaluation of the microstructural gradient along the build direction in electron beam melted Ti6Al-4V alloy. Mater. Sci. Eng. A 744, 182-194 (2019). https:// doi.org/10.1016/j.msea.2018.12.016

26. SAE-International, SAE AS1814-E: Terminology for Titanium Microstructures, pp. 1-17 (2018)

27. C. de Formanoir, S. Michotte, O. Rigo, L. Germain, S. Godet, Electron beam melted Ti-6Al-4V: microstructure, texture and mechanical behavior of the as-built and heat-treated material. Mater. Sci. Eng. A 652, 105-119 (2016). https://doi.org/10.1016/ j.msea.2015.11.052

28. H. Conrad, Effect of interstitial solutes on the strength and ductility of titanium. Prog. Mater. Sci. 26, 123-403 (1981). https:// doi.org/10.1016/0079-6425(81)90001-3

29. S.P. Lynch, S. Moutsos, A brief history of fractography. J. Fail. Anal. Prev. 6, 54-69 (2006). https://doi.org/10.1361/ $154770206 \times 156231$

30. H.M. Tawancy, A. Ul-Hamid, N.M. Abbas, Metallurgical aspects of fracture and fractography, in Practical Engineering Failure Analysis, 1st edn. (CRC Press, Boca Raton, 2004), pp. 397-412. https://doi.org/10.1201/9780203026298.ch10

31. J. Spurrier, J.C. Scully, Fractographic aspects of the stress corrosion cracking of titanium in a methanol/HCL mixture. Corrosion 28, 453-463 (1972)

32. A.L. Pilchak, J.C. Williams, Crystallography of fluted fracture in near- $\alpha$ titanium alloys. Metall. Mater. Trans. A 41A, 22-25 (2010). https://doi.org/10.1007/s11661-009-0091-z

33. D.A. Meyn, E.J. Brooks, Microstructural origin of flutes and their use in distinguishing striationless fatigue cleavage from stresscorrosion cracking in titanium alloys. Fractography Materials Sci. ASTM STP 733, 5-31 (1981)

34. R.C.C. Nixon, D.N. Hawkins, Nature of fluted fracture observed in welds in titanium plate. Mater. Sci. Technol. 5, 288-292 (1989)

35. M. Simonelli, Y.Y. Tse, C. Tuck, On the texture formation of selective laser melted Ti-6Al-4V. Metall. Mater. Trans. A 45A, 2863-2872 (2014). https://doi.org/10.1007/s11661-014-2218-0

Publisher's Note Springer Nature remains neutral with regard to jurisdictional claims in published maps and institutional affiliations. 